Journal of

Accident and

Emergency

Medicine 1994

11, 101-104

\title{
'Bats below the bridge': is a potentially treatable neurovascular disorder being underdiagnosed in accident and emergency departments?
}

\author{
D. HULBERT, S.GABE, D.POTTS, J.A.BALL \& R.TOUQUET
}

Accident and Emergency Department, St Mary's Hospital, Praed Street, London W2 1NY

\section{SUMMARY}

Basilar artery thrombosis (BATS) is a progressive disorder which may present with fluctuating neurological signs of varying degrees of severity. Unless the diagnosis is considered, the confused young patient may be thought to be under the influence of drugs or to be psychologically ill, and indeed the Munchausen syndrome may be considered. The more elderly patient may be diagnosed simply as having had a cerebrovascular accident. This paper presents the cases of three patients admitted through the accident and emergency (A\&E) department of St Mary's Hospital, London with basilar artery thrombosis during a 6-month period. All these patients demonstrated the classic triad for this syndrome of: (1) long tract neurological signs, (2) impaired conscious level, and (3) complex ocular signs.

The concern is that, for patients with more subtle signs, a diagnosis of BATS may not be considered, and a neurological opinion may be thus delayed and no treatment with anticoagulants or thrombolytic agents would be given. Two of the three patients were treated with anticoagulants and improved, while in the third patients anticoagulation was judged inappropriate and the patients died from progression of the disease with respiratory complications.

Key words: basilar artery thrombosis, CVA

\section{INTRODUCTION}

Thrombosis of the distal basilar artery is a condition

Correspondence:

R. Touquet,

Consultant in

Accident and

Emergency,

St. Mary's Hospital,

Praed Street, London

W2 1NY, UK with a poor prognosis. Progression of the disease is related to propagation of the thrombus or resulting emboli. The symptom complex resulting may also be called the 'top of the basilar syndrome' or the 'rostral basilar artery syndrome'.1,2 The patient may present with transient neurological signs due to episodes of neuronal ischaemia secondary to hypoperfusion or embolic phenomena. ${ }^{3}$ The severity of the symptoms is affected to some degree by variations in the anatomy of the circle of Willis. The vertebral arteries are susceptable to injury in neck trauma and in manipulation of the cervical spine. ${ }^{4}$ The most common cause of basilar artery disease is atheroma, but certain other less common but treatable diseases, such as giant cell arteritis and sickle cell disease, should always be considered by the A\&E staff.

The variation in the presenting clinical signs with disease of the posterior cerebral circulation can make detection difficult. Cases may go unrecognized on early presentation, only being diagnosed when signs become more florid. An important differential diagnosis in basilar artery thrombosis is Wernicke's encephalopathy which may present with similar symptoms. However, in the case of Wernicke's disease the onset of symptoms tends to be more gradual and insidious but it is important to identify any history of alcohol misuse.

\section{PATIENTS}

Case 1

The first patient was a 53-year-old man who presented to the A\&E department after falling off his stool at work and noticing a left-sided weakness and difficulty speaking. On examination his blood pressure was $140 / 90$ and he was in sinus rhythm with no carotid bruits or cardiac murmurs. Neurological examination revealed that he was alert and orientated but dysarthric. He had an enlarged right pupil which reacted poorly to light and accommodation, bilateral internuclear ophthalmoplegia (impaired adduction with nystagmus of the abducting eye on congugate lateral gaze), a left lower motor neurone V11 cranial nerve palsy and a poor gag reflex bilaterally. Tone was increased in the left upper limb and both lower 
D. Hulbert et al. limbs with a left pyramidal weakness (grade 4). Reflexes were brisk with bilateral ankle clonus and extensor plantar responses. There was marked left-sided incoordination. Sensory testing was normal.

On the ward over the next $48 \mathrm{~h}$ he became increasingly confused with a fluctuating conscious level. Investigations including full blood count, urea and electrolytes, blood glucose, clotting screen, treponemal serology, ECG and chest radiograph were normal. A CT brain scan performed within $24 \mathrm{~h}$ of admission was normal and excluded a haemorrhagic event. A diagnosis of basilar artery thrombosis was made on clinical grounds. The patients was warfarinized and made an excellent recovery aided by physiotherapy. He was discharged after 4 weeks.

\section{Case 2}

The second patient was a 69-year-old woman whose first symptom was inability to eat her lunch because of difficulty swallowing. She then fell to the floor whilst trying to get out of her chair. Her relatives put her to bed and found her unresponsive some $45 \mathrm{~min}$ later. The only significant past medical history was atrial fibrillation controlled with digoxin. On examination she was in controlled atrial fibrillation with a blood pressure of $130 / 80$ and no evidence of carotid bruits or cardiac murmurs. Neurological examination revealed that she was withdrawing only to deep pain. She had pinpoint pupils and decreased tone and reflexes in all limbs with bilateral extensor plantar responses.

Over the next $24 \mathrm{~h}$ following admission there was some improvement. She obeyed one stage commands but there was some fluctuation in her consciousness level. The following day she developed a right lateral gaze palsy, poor gag reflex bilaterally, was dysarthric, had a left sided ataxic pyramidal weakness and bilateral extensor plantar responses. There was no sensory abnormality. Investigations including full blood count, urea and electrolytes, blood glucose, clotting screen, treponemal serology and chest radiograph were normal. ECG confirmed controlled atrial fibrillation. A CT scan of the brain 7 days after admission showed bilateral thalamic infarcts (see Fig. 1) suggesting an embolic origin within the vertebro-basilar arteries as there was no evidence of infarcts elsewhere in the brain. She was warfarinized, made a good recovery

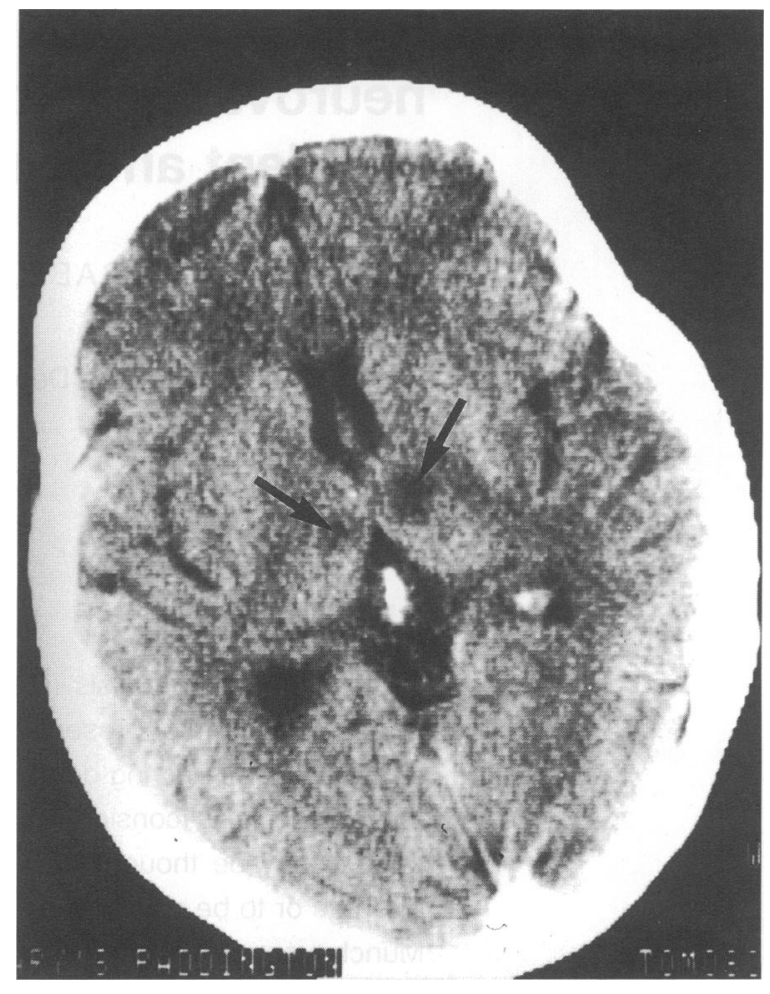

Fig. 1. Computerized tomographic scan of brain of Patient 2 showing bilateral thalamic infarcts (arrows).

\section{Case 3}

The third patient was a 78-year-old man who presented to the A\&E department complaining of gradually worsening vertigo and unsteadiness over a period of 3 days. He also complained of some disturbance of vision. On examination he was hypertensive with a blood pressure of 220/130 $\mathrm{mmHg}$, in sinus rhythm, with no evidence of carotid bruits or cardiac murmurs. Neurological testing revealed poor concentration and memory, dyslexia (but intact letter recognition), visual disorientation (but no hemianopia), a left internuclear ophthalmoplegia and a partial left gaze palsy. Testing of the limbs revealed normal tone and power with brisk reflexes and bilateral flexor plantar responses. There was truncal ataxia but no limb ataxia. Sensory testing was normal. Routine investigations were normal. CT scan of his brain within $24 \mathrm{~h}$ of admission was normal and did not reveal any evidence of haemorrhage. A clinical diagnosis of basilar artery thrombosis was made and he gradually improved. He was not anticoagulated in view of his hypertension and age. He improved enough to be discharged independantly after 4 weeks, but was readmitted 2 weeks later with worsening of his 
Underdiagnosis of BATS previous symptoms and signs and died from a bronchopneumonia.

\section{DISCUSSION}

This accident department saw three patients with basilar artery thrombosis over a period of 6 months (out of 49000 patients a year). The three patients presented with, or developed, symptoms typical of distal basilar artery thrombosis. The finding of eye signs, often complex, associated with pyramidal signs and alteration in the level of consciousness are typical. The findings can be explained by the anatomical relations of the structures supplied by the basilar artery and its branches (Figs 2 \& 3).

Basilar artery thrombosis may produce a wide clinical spectrum of symptoms and signs. A patient may be labelled as a 'completed stroke' and consequently investigated inadequately. The other end of the spectrum includes patients with more subtle signs of confusion, memory disturbance or visual disturbance caused by infarction of the thalamus and/or temporal and occipital lobes. The diagnosis may be suspected on clinical grounds by repeated examinations as some signs may be transient.

Metabolic disorders must be excluded (a blood glucose measurement is mandatory on any patient presenting to the A\&E department with an alteration in consciousness level). A CT scan of the brain is useful to exclude a haemorrhagic event, but may not show an infarction until $48 \mathrm{~h}$ after the event. A Magnetic Resonance Imaging (MRI) scan is more sensitive, will show ischaemic changes earlier with better resolution and improved brainstem imaging, and may show the diagnostic absence of signal void in the basilar artery ${ }^{5,6}$ but its use is limited

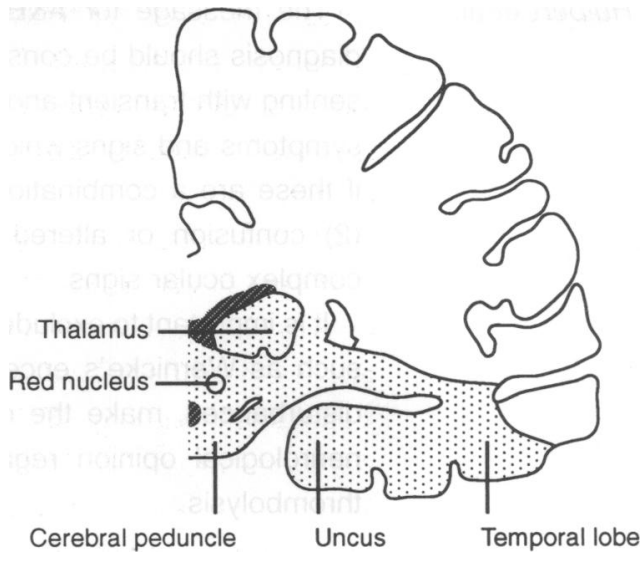

Fig. 3. Coronal section of cerebral hemisphere showing area of posterior cerebral artery (器) supply.

by availability. Vertebral angiography may have complications, particularly in the acute phase, and MRI or MRI angiography may eventually be the investigation of choice. Once the diagnosis of basilar artery thrombosis is made, consideration can be given to anticoagulation. To date there are few controlled trials of its use and the results are controversial. ${ }^{7}$ However, in the future, thrombolysis may be possible and early diagnosis and therapeutic intervention may be crucial. $^{8}$

The actual number of patients presenting with basilar artery thrombosis may be much larger than is realized, with underdiagnosis occuring in those patients with transient signs or in whom the temporal lobe alone is affected. Such patients may have more subtle signs of altered memory or behaviour. Patients may be misdiagnosed as suffering from psychiatric illness, drug side-effects, substance misuse or malingering.

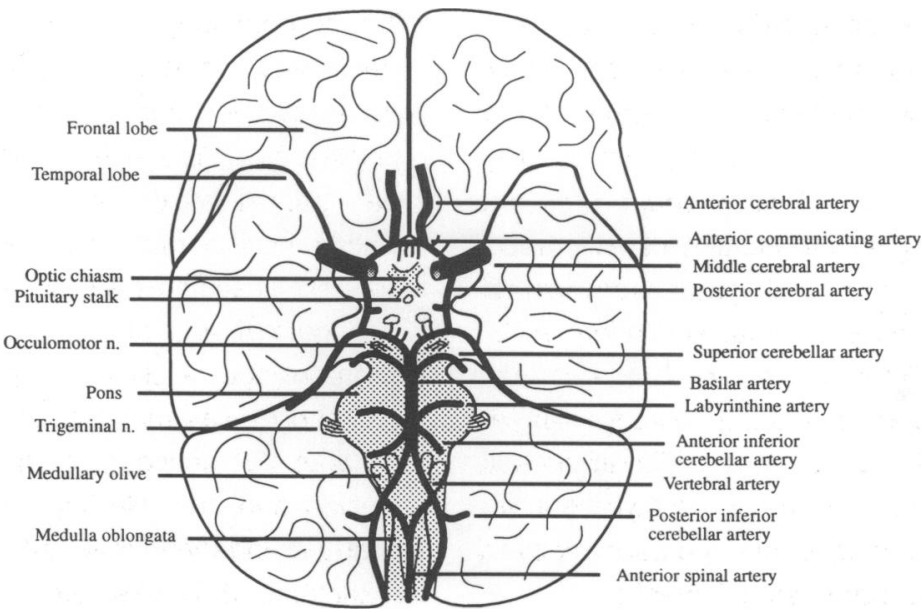

Fig. 2. Diagram to show relation of the basilar artery and its branches to the undersurface of the brain. 
D. Hulbert et al.

The message for A\&E departments is that the diagnosis should be considered in any patient presenting with transient and/or, complex neurological symptoms and signs which are variable, especially if these are a combination of: (1) long tract signs, (2) confusion or altered consciousness, and (3) complex ocular signs.

It is important to exclude other treatable disorders such as Wernicke's encephalopathy or electrolyte disturbances, make the diagnosis and seek early neurological opinion regarding anticoagulation or thrombolysis.

\section{REFERENCES}

1. Caplan L.R. (1980) "Top of the basilar" syndrome. Neurology 30, 72-79.

2. Mehler M.F. (1989) The rostral basilar artery syndrome: Diagnosis, etiology, prognosis. Neurology 39, 9-15.
3. Fisher C.M. (1988) The "Herald hemiparesis" of basilar artery occlusion. Archives of Neurology 45, 1301-1303.

4. Schellhas K.P., Latchaw R.E., Wendling L.R. \& Gold L.H.A. (1980) Vertebrobasilar injuries following cervical manipulation. Journal of the American Medical Association 244, 1450-1453.

5. Barkhof F. \& Valk J. (1988) "Top of the basilar" syndrome: A comparison of clinical and MR finding. Neuroradiology 30, 293-298.

6. Biller J., Yuh W.T., Mitchell G.W., Bruno A. \& Adams H.R.J. (1988) Early diagnosis of basilar artery occlusion using magnetic resonance imaging. Stroke 19(3), 297-306

7. Van Morwijk G. \& Lodder J. (1991) Presumed basilar artery thrombosis and anticoagulation. A survey of 51 cases. Cerebrovasc. Dis 1(4), 227-20.

8. Herderschee D., Limburg M., Hidjra A. \& Koster P.A. (1991) Recombinant tissue plasminogen activator in two patients with basilar artery occlusion. Journal of Neurology, Neurosurgery and Psychiatry 54, 71-73. 